\title{
A Temperature-Insensitive Resonant Pressure Micro Sensor Based on Silicon-on-Glass Vacuum Packaging
}

\author{
Pengcheng Yan ${ }^{1,2}$, Yulan Lu ${ }^{1,2} \mathbb{D}^{\text {, Chao Xiang }}{ }^{1,2}$, Junbo Wang ${ }^{1,2, *}$, Deyong Chen ${ }^{1,2, *}$ and \\ Jian Chen ${ }^{1}$ (1) \\ 1 State Key Laboratory of Transducer Technology, Institute of Electronics, Chinese Academy of Sciences, \\ Beijing 100190, China \\ 2 School of Electronic, Electrical and Communication Engineering, University of Chinese Academy of Sciences, \\ Beijing 100049, China \\ * Correspondence: jbwang@mail.ie.ac.cn (J.W.); dychen@mail.ie.ac.cn (D.C.); Tel.: +86-010-5888-7182 (J.W.); \\ $+86-010-5888-7191$ (D.C.)
}

Received: 9 August 2019; Accepted: 5 September 2019; Published: 7 September 2019

check for updates

\begin{abstract}
This paper presents a temperature-insensitive resonant pressure sensor, which is mainly composed of a silicon-on-insulator (SOI) wafer for pressure measurements and a silicon-on-glass (SOG) cap for vacuum packaging. The variations of pressure under measurement bend the pressure sensitive diaphragm and regulate the intrinsic frequencies of the resonators in the device layer. While, variations of temperature cannot significantly change the intrinsic frequencies of the resonators, due to the SOG cap to offset generated thermal stress. Numerical simulations, based on finite element analysis, were conducted to calculate the residual thermal stress and optimize the sensing structures. Experimental results show that the Q-factors of the resonators are higher than 16,000, with a differential pressure sensitivity of $11.89 \mathrm{~Hz} / \mathrm{kPa}$, a nonlinearity of $0.01 \%$ F.S and a low fitting error of $0.01 \%$ F.S with the pressure varying from $100 \mathrm{kPa}$ to $1000 \mathrm{kPa}$. In particular, a temperature sensitivity of $\sim 1 \mathrm{~Hz} /{ }^{\circ} \mathrm{C}$ was obtained in the range of $-45^{\circ} \mathrm{C}$ to $65^{\circ} \mathrm{C}$, which is one order of magnitude lower than the previously reported counterparts.
\end{abstract}

Keywords: resonant pressure micro sensor; temperature-insensitive; silicon-on-glass cap; MEMS

\section{Introduction}

High-precision silicon-based pressure sensors are increasingly used in the fields of automotive industries and medical electronics and aerospace [1-3]. Compared to other kinds of pressure sensors, such as capacitive pressure sensors, piezoelectric pressure sensors, and piezoresistive pressure sensors, resonant pressure sensors are featured with high accuracies, high resolutions, quasi-digital outputs and long-term stabilities [4]. Resonant pressure sensors are functioned with intrinsic frequency shifts of resonators, due to changes in axial stresses. However, the axial stresses are sensitive to temperature variances for the mismatches of the coefficients of thermal expansion (CTE) between silicon-based sensing elements and packaging materials [5]. Minimizing intrinsic frequency shifts, caused by temperature disturbances, has been a primary research focus for many years [6,7].

Various approaches focusing on packaging materials [8], assembling styles [9], and stress isolation structures $[10,11]$ were proposed to reduce temperature influences. In general, packaging materials with CTE close of that of silicon, such as Pyrex7740 and BOROFLOATß33, were used to seal the dies [8]. Meanwhile, the fragile silicon resonators were generally housed in vacuum chambers rather than over-molded package, which can protect resonators from hostile environments [9]. Moreover, several designs of stress isolation structures were proposed to address the issue of temperature shifts. For instance, an anisotropically etched intermediate layer [10] and a metal interposer structure [11] were 
designed to relax contacting stresses. Previously, we presented a stress isolation structure for resonant pressure sensors, which was realized by mounting the sensor die to the metal substrate, which was fixed at a corner of the sensor through stacks of small silicon dies with silicone adhesive [12]. However, key parameters, such as temperature sensitivity, temperature hysteresis, long-term stability, and offset were still affected by the residual stresses which resulted from anodic bonding and packaging processes.

In this paper, a new type of vacuum packaging, where a silicon-on-glass (SOG) cap was used for reducing the temperature sensitivity of resonant pressure sensors, was proposed. The packaging process bonded a silicon wafer to the glass cap so that the residual thermal stress between the silicon-on-insulator (SOI) wafer and the glass cap could be balanced. The fabrication processes of the pressure sensor, including SOI wafer fabrications and SOG cap fabrications, were thoroughly studied. The effects of the SOG cap on the temperature sensitivity of the sensors were also characterized.

\section{Methodology}

\subsection{Working Principle:}

The proposed resonant pressure sensor is shown in Figure 1a, which mainly consists of a pressure sensitive diaphragm (length: $5000 \mu \mathrm{m}$, width: $5000 \mu \mathrm{m}$ ) in the handle layer, a pair of H-shaped doubly-clamped resonators (length: $1400 \mu \mathrm{m}$, width: $20 \mu \mathrm{m}$, thickness: $40 \mu \mathrm{m}$ ) in the device layer, and an SOG cap with a cavity (length: $5000 \mu \mathrm{m}$, width: $5000 \mu \mathrm{m}$, thickness: $100 \mu \mathrm{m}$ ) in the glass layer. The silicon-glass bonded wafer could reduce the thermal mismatches between the SOI die and the glass wafer during anodic bonding [13-15]. The resonators are located in the relevant middle and the edge of the square diaphragm, which are named "central beam", and "side beam", respectively. In operation, with the deflections of the pressure-sensitive diaphragm, caused by pressure under measurement, the direction of the intrinsic resonant frequency shifts of the two resonators is opposite each other, as shown in Figure 1c. Thus, the differential outputs can be used to minimize the temperature disturbance and amplify the sensitivities of pressure measurement. Electromagnetic excitation/electromagnetic detection was used in this paper to measure the resonant frequencies of the resonators, as shown in Figure $1 \mathrm{~d}$. The detailed detection principle is that the resonator, which is perpendicular to a static magnetic field and carries an AC current, experiences a Lorentz force, drives the resonant beam into vibration, and the resulting vibration produces a magnetic induction voltage, that we can use to obtain the frequency of the resonator by detecting the voltage signal.

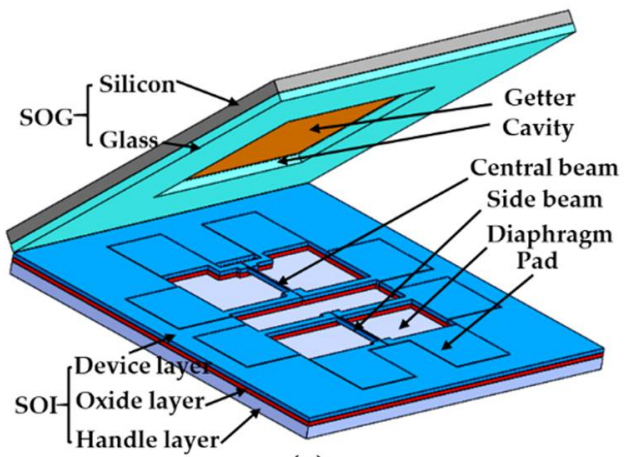

(a)

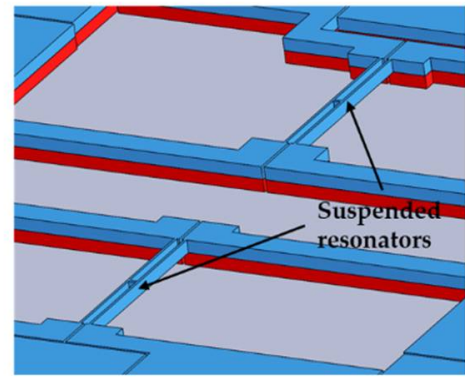

(b)

Figure 1. Cont. 


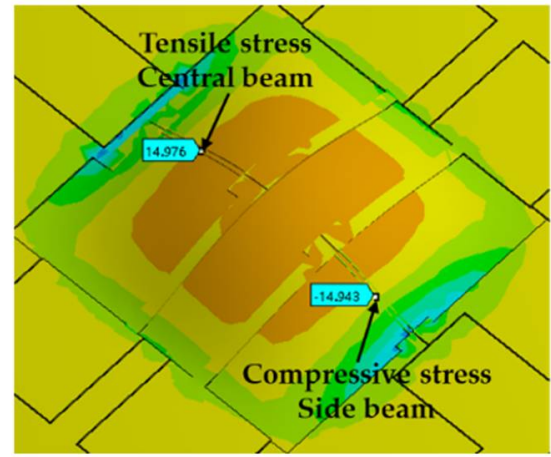

(c)

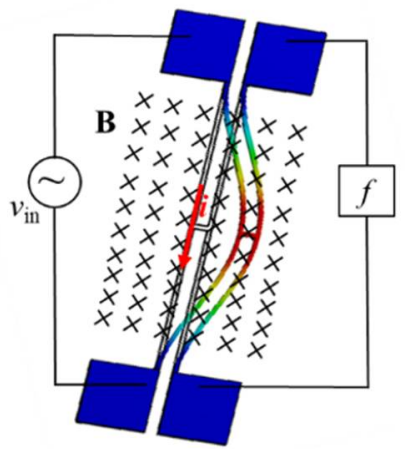

(d)

Figure 1. (a) Schematic of the resonant pressure sensor, including an silicon-on-insulator (SOI) wafer and an silicon-on-glass (SOG) cap. In the SOI wafer, there is a square pressure sensitive diaphragm in the handle layer, two H-shaped doubly-clamped resonators in the device layer. The SOG cap with a cavity, sputtered with the getter material, is used to form a vacuum packaging for the resonators and reduce the temperature sensitivity of the resonant pressure sensor; (b) Zoom in for the key portion of Figure 1a; (c) Pressure under measurements causes the deformation of the pressure-sensitive diaphragm, leading to frequency shift of the resonators; (d) Electromagnetic excitation/electromagnetic detection, the resonator experiences a Lorentz force to excite the beam into vibration, and the resulting vibrations are sensed by detecting the magnetically induced voltage, developed across the beam.

\subsection{Thermomechanical Mechanism and Simulations:}

Due to the mismatches of CTE, between silicon-based sensing elements and packaging materials, temperature changes will generate residual thermal stress at the bonding interface. Figure 2a shows that the glass contracted faster than silicon while the temperature dropped from bonding temperature to ambient working temperature. According to thermomechanical mechanisms [16], the residual thermal stress at the interface between silicon and glass should be,

$$
\sigma=\frac{E_{2}\left(\alpha_{2}-\alpha_{1}\right) \Delta T}{1+\frac{E_{2} t_{2}}{E_{1} t_{1}}}
$$

where $E_{1}, E_{2}$ are Young's modulus, $t_{1}, t_{2}$ are the thickness and $\alpha_{1}, \alpha_{2}$ are coefficients of thermal expansion of glass and silicon, respectively. A silicon wafer without features was bonded to the glass substrate to offset the thermal stress. Assuming that CTE of the bi-layer is $\alpha_{3}$, it is closer to $\alpha_{2}$ than $\alpha_{1}$. In addition, the glass layer was thinned using chemical mechanical planarization to make $E_{2} t_{2} \gg E_{1} t_{1}$ so that the residual thermal stress could be further reduced, as shown in Figure $2 b$.

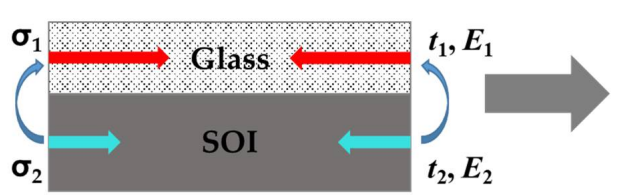

(a)

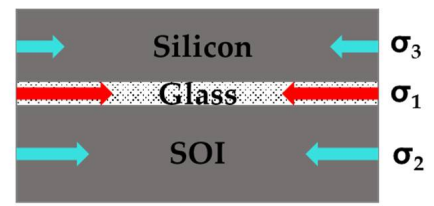

(b)

Figure 2. Schematic of multi-layer structures: the SOI wafer bonded with (a) the pure glass cap and (b) the SOG cap.

Numerical simulations, based on finite element analysis (FEA), were conducted to reveal the relationships between the residual thermal stresses and the intrinsic frequency shifts of the resonators, in order to optimize the sensor structures. More specifically, thermal stresses, based on a multi-mode of steady-state thermal analysis and static structural analysis, were first calculated. The materials used in simulations are listed in Table 1. To reduce the master degrees of freedom of the entire model, two 
element sizes were used in these simulations. A meshing size of $100 \mu \mathrm{m}$ was used to mesh the entire body, except the resonant beams, which were meshed in an element size of $10 \mu \mathrm{m}$. Then, the thermal stresses were calculated as the temperature dropped from bonding temperature $\left(350^{\circ} \mathrm{C}\right)$ to ambient working temperature $\left(-55^{\circ} \mathrm{C} \sim 85^{\circ} \mathrm{C}\right)$. Finally, a modal analysis was conducted to obtain the intrinsic resonant frequencies by using the thermal stresses as loads.

Table 1. Properties of materials used in finite element method.

\begin{tabular}{cccc}
\hline Items & & Silicon & BF33 \\
\hline Young's modulus (GPa) & & 165 & 64 \\
Density (g/cm3) & & 2.33 & 2.23 \\
Poisson's ratio & & 0.28 & 0.2 \\
\hline & $20{ }^{\circ} \mathrm{C}$ & 2.46 & 3.25 \\
$\mathrm{CTE}\left(\mathrm{ppm} /{ }^{\circ} \mathrm{C}\right)$ & $250^{\circ} \mathrm{C}$ & 3.61 & 3.25 \\
& $500^{\circ} \mathrm{C}$ & 4.15 & 3.5 \\
\hline
\end{tabular}

To analyze the side effects of temperature disturbances, three types of packaging methods were modelled and compared, where the intrinsic frequency shifts of the two resonators as functions of temperature variances, were calculated. Figure $3 \mathrm{a}-\mathrm{c}$ represent the residual thermal stress distributions of the SOI wafers after anodic bonding. The equivalent stresses in the SOI wafer bonded with the pure glass cap (500 $\mu \mathrm{m}$ in thickness), the SOG cap I (300 $\mu \mathrm{m}$-thick silicon and $500 \mu \mathrm{m}$-thick glass) and the SOG cap II (300 $\mu \mathrm{m}$-thick silicon and $50 \mu \mathrm{m}$-thick glass) were noticed to decrease successively, which indicates that the thermal stress could be reduced by adjusting the thickness of the glass substrate. The resonant frequencies of the resonators were calculated at a pressure of $100 \mathrm{kPa}$ and a temperature of $22{ }^{\circ} \mathrm{C}$ (see Figure 3d), where the devices capped with the SOG cap II exhibit minimum frequency shift among the three types of devices. Furthermore, the temperature sensitivities of the three types of devices were quantified as $18.02 \mathrm{~Hz} /{ }^{\circ} \mathrm{C}, 8.75 \mathrm{~Hz} /{ }^{\circ} \mathrm{C}$, and $0.42 \mathrm{~Hz} /{ }^{\circ} \mathrm{C}$, respectively, under the pressure of $100 \mathrm{kPa}$ (see Figure 3e). Thus, an optimized thickness of $50 \mu \mathrm{m}$ of the glass substrate was chosen to seal the die.

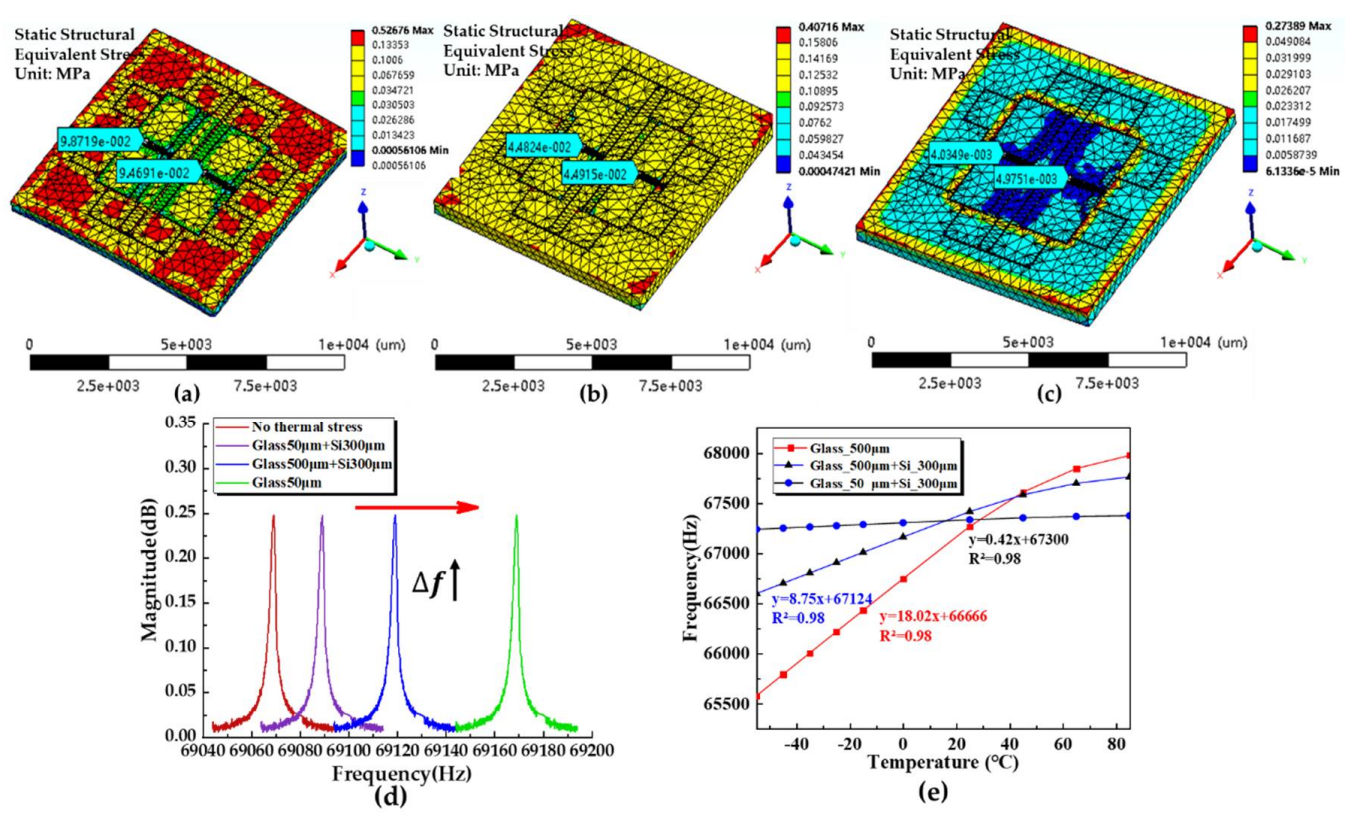

Figure 3. Simulation results of finite element analysis based on ANSYS: (a-c) Distributions of residual thermal stresses for the sensor chips capped with the pure glass cap (500 $\mu \mathrm{m}$ in thickness), the SOG cap I (300 $\mu \mathrm{m}$-thick silicon and $500 \mu \mathrm{m}$-thick glass) and the SOG cap II (300 $\mu \mathrm{m}$-thick silicon and $50 \mu \mathrm{m}$-thick glass) from left to right; (d) Intrinsic frequency shifts of three types of senor chips in response to applied pressure; (e) Intrinsic frequency shifts of three types of senor chips in response to temperature variation. 


\section{Fabrication}

The fabrication processes of the sensor, include the fabrications of the SOI wafer and the SOG cap. A 4 inch SOI wafer ((100) plane, $<100>$ oriented, p-type) with a device layer of $40 \mu \mathrm{m}$, a buried SiO2 layer of $2 \mu \mathrm{m}$ and a handle layer of $300 \mu \mathrm{m}$ was utilized. Three photolithographic and deep reactive iron etch (DRIE) steps were used to form the resonators, the pressure-sensitive diaphragm and the through silicon vias (TSVs). Firstly, the SOI wafer was cleaned by a standard wafer cleaning process. Then, a $140 \mu \mathrm{m}$-thick pressure sensitive diaphragm and $300 \mu \mathrm{m}$-deep TSV holes were etched on the handle layer by DRIE (see Figure $4 b$ ). To realize the uniform thickness of the diaphragm, a patterned $\mathrm{ZnO}$ film of $1000 \AA$ and $5.4 \mu \mathrm{m}$-thick positive photoresist (AZ4620) were used as aligned masks in DRIE [17]. Afterwards, the resonators in the device layer were fabricated by DRIE with a patterned photoresist mask and the sacrificial layer release-etch (see Figure 4c,d).

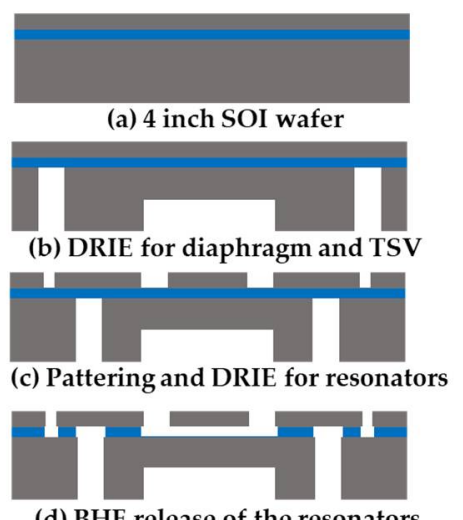

(d) BHF release of the resonators

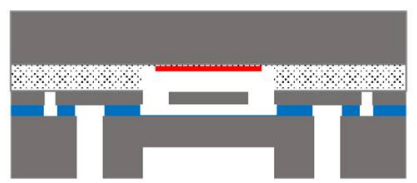

(i) SOI-SOG anodic bonding

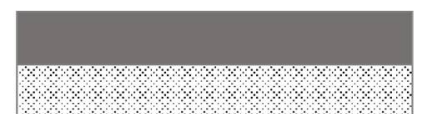

(e) Si-glass anodic bonding

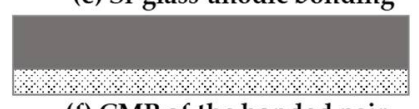

(f) CMP of the bonded pair

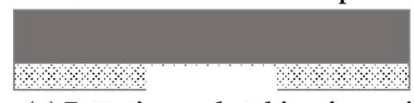

(g) Pattering and etching for cavity

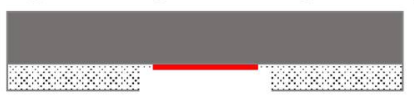

(h) Sputtering the getter material

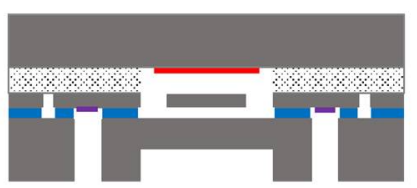

(j) Sputtering $\mathrm{Cr} / \mathrm{Au}$ as electrodes

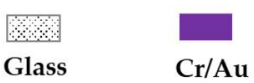

Figure 4. Fabrication process of the proposed resonant pressure micro sensor: (a-d) Fabrication of the SOI wafer; (e-h) Fabrication of the SOG cap; (i) SOI-SOG anodic bonding; (j) Fabrication of electrodes.

For the SOG cap, the process started with anodic bonding between a $300 \mu \mathrm{m}$-thick Si wafer and a $300 \mu \mathrm{m}$-thick glass wafer, under a voltage of $800 \mathrm{~V}$ and a surrounding temperature of $350^{\circ} \mathrm{C}$ (see Figure $4 \mathrm{e}$ ). Then the chemical mechanical planarization was used to thin the glass layer to the final thickness of $50 \mu \mathrm{m}$ (see Figure 4f). Then the compound substrate was thoroughly cleaned to remove organic residues and other particulates. The getter cavity was defined by etching about $35 \mu \mathrm{m}$-depth cavity, where a sputtered $\mathrm{Cr} / \mathrm{Au}$ film of $300 \AA / 3000 \AA$ and $5.4 \mu$ m-thick photoresist functioned as compound masks (see Figure $4 \mathrm{~g}$ ). The depth of the cavity defined the distance between the resonators and the cap, which affected the temperature sensitivity as well. After stripping the mask for the glass cavity, a Ti/Au film of $5000 \AA / 300 \AA$ was sputtered inside the cavity as the getter layer, which was used to absorb the gases generated during anodic bonding, ensuring that the resonators work at a high vacuum. (Figure $4 \mathrm{~h}$ ). The resonators were sealed in the vacuum chamber by bonding the SOG wafer with the fabricated SOI wafer at a low pressure of about $0.1 \mathrm{~Pa}$ under a voltage of $250 \mathrm{~V}$ and a temperature of $350{ }^{\circ} \mathrm{C}$ (see Figure 4i). Finally, a Cr/Au film was sputtered into the TSVs as electrodes.

The proposed resonant pressure micro sensor was successfully made by bulk-silicon micromachining technology. As shown in Figure 5a, the etching depth and the undercut of the fabricated SOG wafer were measured by scanning electron microscopy (SEM), where a $50.8 \mu \mathrm{m}$-deep 
cavity and an undercut of $79.4 \mu \mathrm{m}$ were found. Figure $5 b, c$ shows the cross section and the top view of a suspended resonator, respectively, where an undercut of $16.6 \mu \mathrm{m}$ was found on the anchor. The lateral etching in the oxide film was so small, that its effects on the rest of the structure could be ignored. Figure $5 \mathrm{~d}$ shows the image of a prototype sensor with a dimension of $10 \mathrm{~mm} \times 10 \mathrm{~mm}$. Figure 5e shows the package prototypes of a sensor unit after wafer dicing.

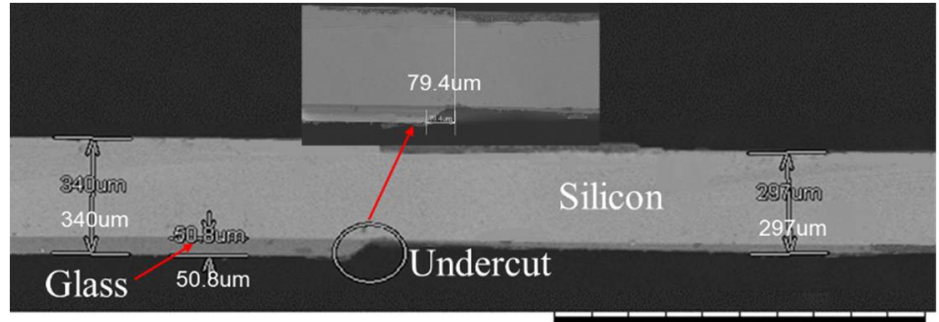

(a)

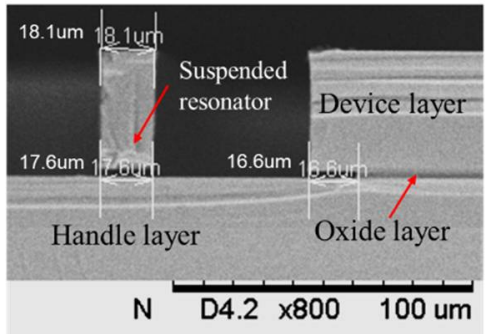

(b)

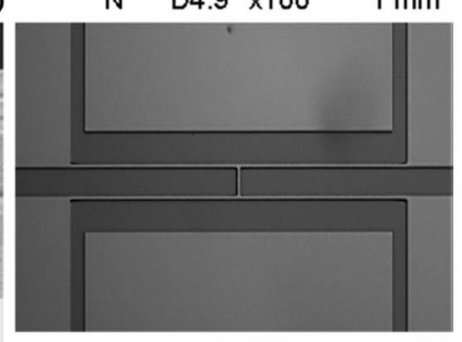

(c)

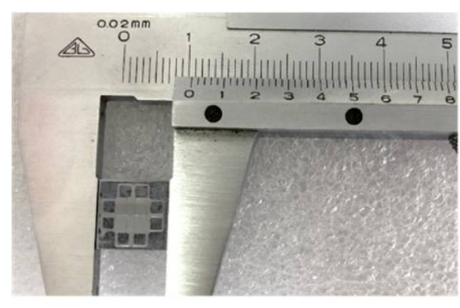

(d)

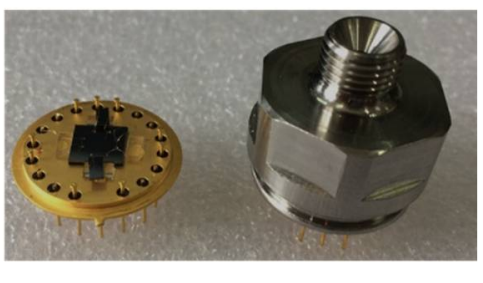

(e)

Figure 5. (a) The scanning electron microscopy (SEM) image of the cross section of the SOG cap; (b) Cross section of the suspended resonator with an undercut of $16.6 \mu \mathrm{m}$ on the anchor; (c) Image of the top view of the resonator; (d) Image of a prototype sensor with a dimension of $10 \mathrm{~mm} \times 10 \mathrm{~mm}$; (e) Image of packaged prototype of the resonant pressure micro sensor.

\section{Characterizations}

An E5061B Network Analyzer (Agilent, USA) was utilized to obtain the frequency response of the fabricated sensor ship in an open-loop scanning manner (see Figure 6a). The resonant frequencies of the resonators were quantified as $\sim 67,736 \mathrm{~Hz}$ with the phase shift of $\sim 180^{\circ}$ and Q-factors of 16,589 , which indicated the reliability of the triple-stack anodic bonding of SOI-SOG, as shown in Figure $6 \mathrm{~b}$. In addition, multiple cycles of open-loop testing were conducted to obtain the shifts of the resonant frequencies (see Figure 6c,d). By comparing the pressure sensors with glass or SOG caps, it was found that the resonant pressure sensor with the SOG caps produced less shifts of the resonant frequencies when the testing cycles were increased from one to six, which demonstrates that the proposed resonant pressure sensors are more stable than the pressure sensors with glass caps.

Furthermore, a closed-loop self-oscillating system was developed to characterize the performances of the proposed sensors, as shown in Figure 7a. The system mainly includes, an amplifier circuit, multiple filter circuit, drive buffer circuit, and automatic gain control (AGC) circuit. The induced voltage of the resonators was amplified by an instrument amplifier, and then the signal was sent to the driving end of the resonator after being attenuated and buffered. An automatic gain control circuit, including a band-pass filter, a comparator, a rectifier and a junction field effect transistor was developed to maintain the stable vibrations of the resonators. The intrinsic frequencies of the resonators were detected under various pressure and temperature, controlled by a pressure calibrator (PPC4, Fluck Co., Phoenix, AZ, USA) and a temperature and humidity chamber (SH-241, ESPEC Co., Osaka, Japan). 


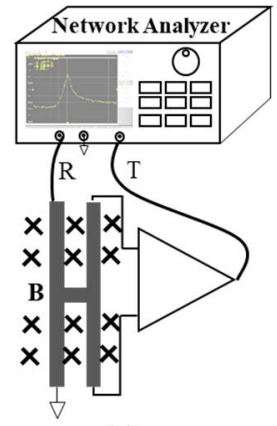

(a)

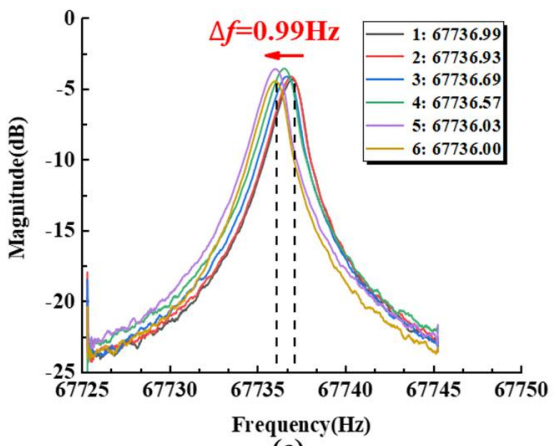

(c)

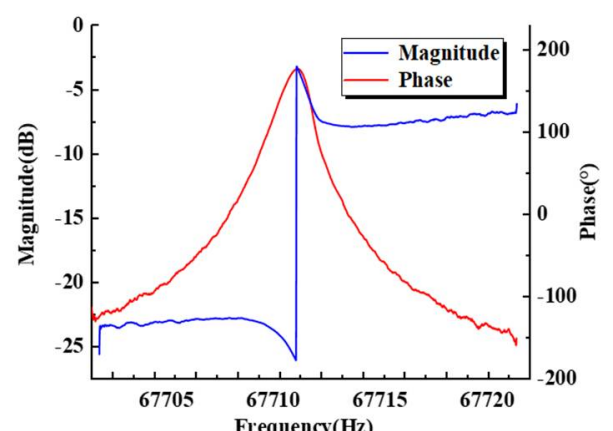

(b)

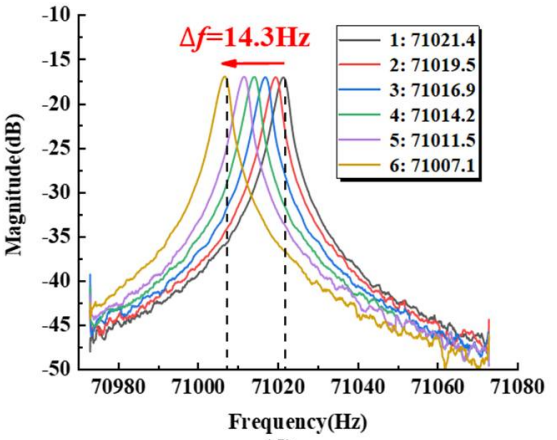

(d)

Figure 6. (a) Schematic of the open-loop platform for the resonators; (b) open loop result of the sensor, recording a beam resonant frequency of $\sim 67736 \mathrm{~Hz}$ (quality factor of 16589); multiple cycles of frequency responses of resonators based on SOG, (c) glass, and (d) vacuum packing.

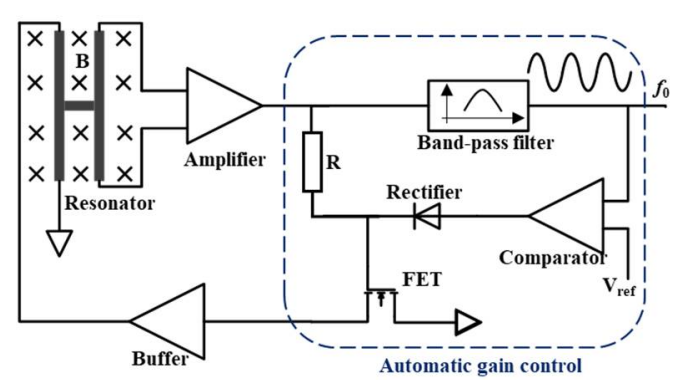

(a)

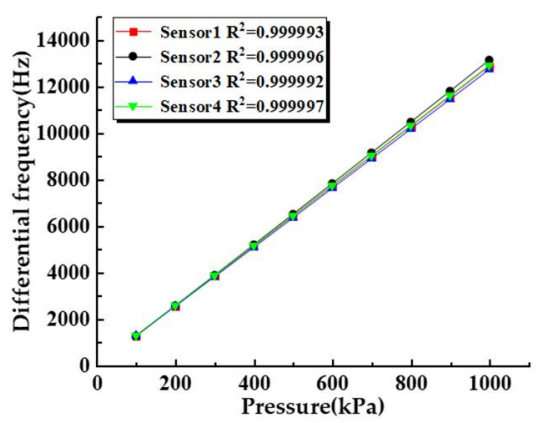

(c)

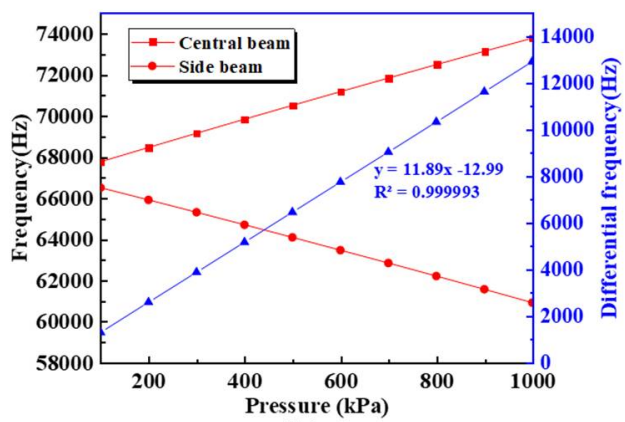

(b)

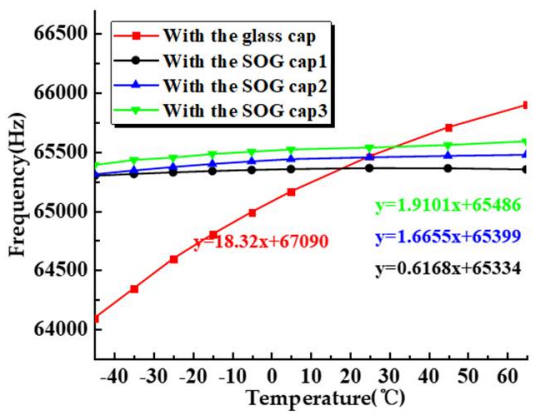

(d)

Figure 7. Cont. 


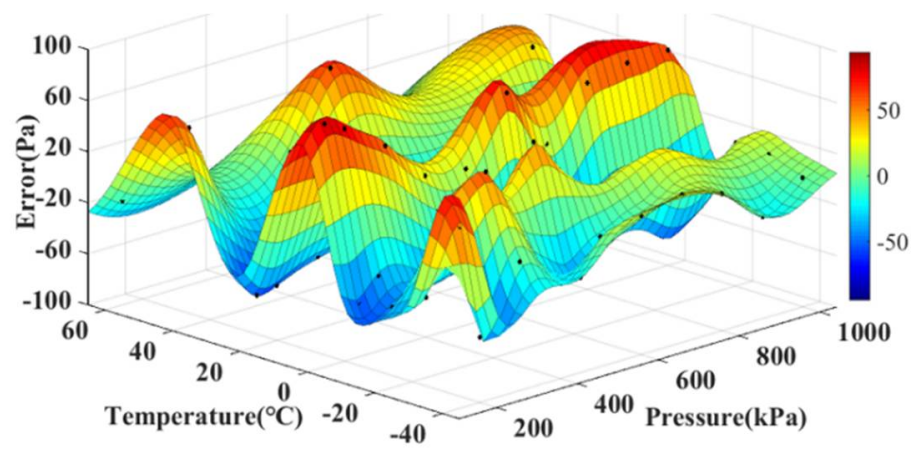

(e)

Figure 7. Characterization results: (a) Schematic of the self-oscillation system including an amplifier module, a drive buffer module and an automatic gain control (AGC) circuit; (b) Pressure sensitivities of the sensors with the pressure varying from $100 \mathrm{kPa}$ to $1000 \mathrm{kPa}$ at room temperature; (c) Differential pressure sensitivities of four pressure sensors, indicating a nonlinearity of $0.01 \%$ within the pressure range of $100 \mathrm{kPa}$ to $1000 \mathrm{kPa}$; (d) Temperature sensitivity of the pressure sensor with different caps under an applied pressure of $100 \mathrm{kPa}$; (e) Fitting errors of the proposed sensor within the full pressure range of $100 \mathrm{kPa}$ to $1000 \mathrm{kPa}$ and temperature range of $-45^{\circ} \mathrm{C}$ to $65^{\circ} \mathrm{C}$.

In this study, the devices were characterized with a pressure range from $100 \mathrm{kPa}$ to $1000 \mathrm{kPa}$ (one point per $100 \mathrm{kPa}$ ) and a temperature range from $-45^{\circ} \mathrm{C}$ to $65{ }^{\circ} \mathrm{C}$ (one point per $10{ }^{\circ} \mathrm{C}$ or $20^{\circ} \mathrm{C}$ ). Figure $7 \mathrm{~b}$ shows differential pressure sensitivities of the resonators, which were quantified as $11.89 \mathrm{~Hz} / \mathrm{kPa}$ with correlation coefficient of 0.999993 . Figure $7 \mathrm{c}$ shows the plot of the differential pressure sensitivities of four sensors, indicating a nonlinearity of $0.01 \%$ within the pressure range of $100 \mathrm{kPa}$ to $1000 \mathrm{kPa}$. By comparing the pressure sensors with glass or SOG caps, it was found that the temperature sensitivities of the sensors decreased from $18.32 \mathrm{~Hz} /{ }^{\circ} \mathrm{C}$ to $\sim 1 \mathrm{~Hz} /{ }^{\circ} \mathrm{C}$, as shown in Figure $7 \mathrm{~d}$. Polynomial surface fitting with the calibration data was conducted to obtain the difference (error) between the experimental results and the compensated pressure value [18]. Figure 7e shows the fitting result of the proposed resonant pressure micro sensor in the full pressure scale (100 kPa 1000 kPa) and temperature range $\left(-45^{\circ} \mathrm{C}\right.$ to $\left.65^{\circ} \mathrm{C}\right)$. It was observed that the errors were within $\pm 90 \mathrm{~Pa}$ with an accuracy better than $\pm 0.01 \%$ F.S $(1000 \mathrm{kPa})$. As a consequence, it could be concluded that temperature-insensitive resonant pressure sensor could be realized by the SOG vacuum packaging.

\section{Conclusions}

A temperature-insensitive resonant pressure sensor based on the SOG vacuum packaging is presented in this paper. The SOG cap not only provides hermetic sealing for the resonators but offsets the residual thermal stress between the SOI wafer and the glass cap. The experimental results shows the quality factors of the resonators were higher than 16,000 with a differential pressure sensitivity of $11.89 \mathrm{~Hz} / \mathrm{kPa}$. Further characterizations based on a closed-loop self-oscillating system indicate that the proposed sensor feature with low nonlinearity within $0.01 \%$ F.S and low fitting errors within $0.01 \%$ F.S under the pressure range of $100 \mathrm{kPa}$ to $1000 \mathrm{kPa}$ in temperature range of $-45^{\circ} \mathrm{C}$ to $65^{\circ} \mathrm{C}$. In addition, with the SOG cap, the temperature sensitivity of the sensor dropped from $18.32 \mathrm{~Hz} /{ }^{\circ} \mathrm{C}$ to $\sim 1 \mathrm{~Hz} /{ }^{\circ} \mathrm{C}$. The effective structure could be further developed to improve the performance of the resonant pressure sensor.

Author Contributions: J.W., D.C. defined the parameters of the project; P.Y. and C.X. designed the device; P.Y. and Y.L. fabricated the device; P.Y. and C.X. characterized the devices; P.Y., Y.L., and J.C. wrote the manuscript.

Funding: This work was supported by the National Key Research and Development Program (2018YFF01010400 and 2018YFB2002302) and the National Natural Science Foundation of China (Grant No. 61825107, U1930206 and 61431019).

Conflicts of Interest: The authors declare no conflict of interest. 


\section{References}

1. Ikeda, K.; Kuwayama, H.; Kobayashi, T.; Watanabe, T.; Nishikawa, T.; Yoshida, T.; Harada, K. Silicon pressure sensor integrates resonant strain gauge on diaphragm. Sens. Actuators A Phys. 1990, 21, 146-150. [CrossRef]

2. Welham, C.J.; Greenwood, J.; Bertioli, M.M. A high accuracy resonant pressure sensor by fusion bonding and trench etching. Sens. Actuators A Phys. 1999, 76, 298-304. [CrossRef]

3. Chen, D.; Li, Y.; Liu, M.; Wang, J. Design and experiment of a laterally driven micromachined resonant pressure sensor for barometers. Procedia Eng. 2010, 5, 1490-1493. [CrossRef]

4. Corman, T.; Enoksson, P.; Stemme, G. Low-pressure-encapsulated resonant structures with integrated electrodes for electrostatic excitation and capacitive detection. Sens. Actuators A Phys. 1998, 66, 160-166. [CrossRef]

5. Melamud, R.; Hopcroft, M.; Jha, C.; Kim, B.; Chandorkar, S.; Candler, R.; Kenny, T.W. Effects of stress on the temperature coefficient of frequency in double clamped resonators. In Proceedings of the 13th International Conference on Solid-State Sensors, Actuators and Microsystems, 2005 (TRANSDUCERS '05), Seoul, Korea, 5-9 June 2005; pp. 392-395.

6. Hao, Y.; Yuan, W.; Xie, J.; Shen, Q.; Chang, H. Design and verification of a structure for isolating packaging stress in SOI MEMS devices. IEEE Sens. J. 2016, 17, 1246-1254. [CrossRef]

7. Li, A.; Zhang, C.; Wang, H.; He, Y.; Sun, D.; Wang, L.; Du, X.; Gu, D. Design of temperature-immunization system packaging for the resonant pressure sensor. Mod. Phys. Lett. B 2017, 31, 1750085. [CrossRef]

8. Rogers, T.; Kowal, J. Selection of glass, anodic bonding conditions and material compatibility for silicon-glass capacitive sensors. Sens. Actuators A Phys. 1995, 46, 113-120. [CrossRef]

9. Lee, J.S.; Faheem, F.; Kim, J.F.; Jung, J.D. MEMS packaging technology using a cavity structure for mass production. In Proceedings of the International Conference on Electronic Materials \& Packaging, Daejeon, Korea, 19-22 November 2007.

10. Offereins, H.; Sandmaier, H.; Folkmer, B.; Steger, U.; Lang, W. Stress free assembly technique for a silicon based pressure sensor. In Proceedings of the 1991 International Conference on Solid-State Sensors and Actuators (Transducer'91), San Francisco, CA, USA, 24-27 June1991; pp. 986-989.

11. Marinis, T.F.; Soucy, J.W.; Hanson, D.S. Isolation of MEMS devices from package stresses by use of compliant metal interposers. In Proceedings of the 56th Electronic Components and Technology Conference 2006, San Diego, CA, USA, 30 May-2 June 2006.

12. Li, Y.; Chen, D.; Wang, J. Vacuum adhesive bonding and stress isolation for MEMS resonant pressure sensor package. Mater. Sci. Forum 2011, 694, 896-900. [CrossRef]

13. Dinh, T.; Phan, H.P.; Kashaninejad, N.; Nguyen, T.K.; Dao, D.V.; Nguyen, N.T. An on-chip SiC MEMS device with integrated heating, sensing, and microfluidic cooling systems. Adv. Mater. Interfaces 2018, 5, 1800764. [CrossRef]

14. Dinh, T.; Phan, H.-P.; Nguyen, T.-K.; Balakrishnan, V.; Cheng, H.-H.; Hold, L.; Lacopi, A.; Nguyen, N.-T.; Dao, D.V. Unintentionally doped epitaxial 3C-SiC (111) nanothin film as material for highly sensitive thermal sensors at high temperatures. IEEE Electron. Device Lett. 2018, 39, 580-583. [CrossRef]

15. Phan, H.-P.; Cheng, H.-H.; Dinh, T.; Wood, B.; Nguyen, T.-K.; Mu, F.; Kamble, H.; Vadivelu, R.; Walker, G.; Hold, L. Single-crystalline 3C-SiC anodically bonded onto glass: An excellent platform for high-temperature electronics and bioapplications. ACS Appl. Mater. Interfaces 2017, 9, 27365-27371. [CrossRef] [PubMed]

16. Senturia, S.D. Microsystem Design; Springer Science \& Business Media: Berlin, Germany, 2007.

17. Xie, B.; Xing, Y.; Wang, Y.; Chen, D.; Wang, J. Vacuum-packaged resonant pressure sensor with dual resonators for high sensitivity and linearity. Procedia Eng. 2015, 120, 194-199. [CrossRef]

18. Li, Y.; Wang, J.; Luo, Z.; Chen, D.; Chen, J. A resonant pressure microsensor capable of self-temperature compensation. Sensors 2015, 15, 10048-10058. [CrossRef] [PubMed]

(C) 2019 by the authors. Licensee MDPI, Basel, Switzerland. This article is an open access article distributed under the terms and conditions of the Creative Commons Attribution (CC BY) license (http://creativecommons.org/licenses/by/4.0/). 